\title{
Minimal difference between fractionated and single-fraction exposure in a murine model of radiation necrosis
}

\author{
Andrew J. Boria ${ }^{1}$ and Carlos J. Perez-Torres ${ }^{1,2^{*}}$ (i)
}

\begin{abstract}
Purpose: Despite the success of fractionation in clinical practice to spare healthy tissue, it remains common for mouse models used to study the efficacy of radiation therapy to use minimal or no fractionation. The goal of our study was to create a fractionated mouse model of radiation necrosis that we could compare to our single fraction model.

Methods: Precision X-Ray's X-Rad 320 cabinet irradiator was used to irradiate the cerebrum of mice with four different fractionation schemes, while a 7 T Bruker magnetic resonance imaging (MRI) scanner using T2 and postcontrast T1 imaging was used to track the development of radiation necrosis over the span of six weeks.

Results: All four fractionation schemes with single fraction equivalent doses (SFED) less than 50 Gy for the commonly accepted alpha/beta ratio ( $\alpha / \beta)$ value of $2-3$ Gy produced radiation necrosis comparable to what would be achieved with single fraction doses of 80 and $90 \mathrm{~Gy}$. This is surprising when previous work using single fractions of $50 \mathrm{~Gy}$ produced no visible radiation necrosis, with the results of this study showing fractionation not sparing brain tissue as much as expected.

Conclusion: Further interpretation of these results must take into consideration other studies which have shown a lack of sparing when fractionation has been incorporated, as well as consider factors such as the use of large doses per fraction, the time between fractions, and the limitations of using a murine model to analyze the human condition.
\end{abstract}

Keywords: Fractionation, Mouse model, MRI, Radiation biology, Radiation necrosis

\section{Introduction}

Radiation therapy is essential to cancer treatment, with approximately $50 \%$ of cancer patients receiving radiation therapy and radiation contributing toward $40 \%$ of the curative treatments of the disease [1]. Fractionated radiation therapy is the most prominent technique for treating cancer with radiation [2] due primarily to fractionation allowing for the selective sparing of healthy tissue $[1,3]$. Fractionation thus serves clinically to reduce the complications attributed to radiation therapy [4-7].

Though fractionation is the established approach clinically, it remains common in preclinical studies to

\footnotetext{
* Correspondence: cperezto@purdue.edu

${ }^{1}$ School of Health Sciences, Purdue University, 550 Stadium Mall Drive, Hampton Hall 1263A, West Lafayette, IN 47907, USA

${ }^{2}$ Purdue University Center for Cancer Research, Purdue University, West Lafayette, IN, USA
}

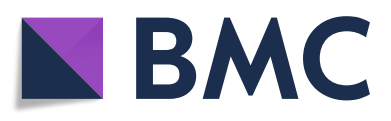

(c) The Author(s). 2019 Open Access This article is distributed under the terms of the Creative Commons Attribution 4.0 International License (http://creativecommons.org/licenses/by/4.0/), which permits unrestricted use, distribution, and reproduction in any medium, provided you give appropriate credit to the original author(s) and the source, provide a link to the Creative Commons license, and indicate if changes were made. The Creative Commons Public Domain Dedication waiver (http://creativecommons.org/publicdomain/zero/1.0/) applies to the data made available in this article, unless otherwise stated. high single fraction doses [8-12]. This unfractionated approach has practical advantages such as a shorter time commitment and avoiding potential confounds due to the potentially limited reproducibility of positioning for focal treatments. Even so, ideally animal models of radiation-induced injury should be performed with fractionated regimes to ensure that the radiation exposure is as human-like as possible.

We have recently published a mouse model of radiation necrosis generated with a large single fraction treatment [13]. Based on the logic above, our goal was to create a fractionated mouse model in order to treat mice in a way more similar to how humans are treated with radiation. Our hypothesis was that fractionation would provide a noticeable level of sparing to healthy 
tissue as seen in patients. However, the level of sparing was found to be minimal, with fractionation schemes predicted not to cause radiation necrosis based on our previous findings [13] instead causing radiation necrosis almost as severe as observed with single fraction doses of equal total dose.

\section{Materials and methods}

All animal experiments were approved by the Purdue Animal Care and Use Committee. The general experimental framework included irradiation followed by MRI to track radiation necrosis lesion progression and finally post-mortem validation with histology.

\section{Setup and treatment}

Irradiation was performed as previously described [13] so that our fractionated treatments are comparable to the single fraction data from that publication. Briefly, an X-Rad 320 (Precision X Ray, North Branford, CT) preclinical cabinet irradiator was used to deliver partial cerebrum doses to mice via a field $0.5 \mathrm{~cm}$ by $0.5 \mathrm{~cm}$ such that a single hemisphere was irradiated at a dose rate of about 2 Gy per minute. Female $8-9$ week old BALB/c (Harlan, Indianapolis, IN) were irradiated once a day Monday through Friday as is usually done in the clinic.

\section{Fractionation}

Four different radiation fractionation schemes were used: 5 fractions of $20 \mathrm{~Gy}, 10$ fractions of $10 \mathrm{~Gy}, 5$ fractions of $18 \mathrm{~Gy}$, and 10 fractions of $9 \mathrm{~Gy}$. We chose not to do more than 10 fractions over two weeks because in our previous work onset of pathology occurred at 2 or 3 weeks for 100 and 90 Gy in a single fraction respectively [13]. We were concerned that further protraction might lead to overlap of treatment and lesion onset. Based on the linear-quadratic model, the biologically effective dose (BED) and single fraction equivalent dose (SFED) were calculated for all four schemes and are included in Table 1 based off the commonly assumed alpha/beta ratio $(\alpha / \beta)$ for early and late responding tissue [14]. For both equations, $\mathrm{n}$ is the fraction number, $\mathrm{d}$ is the dose per fraction in Gy, and $\alpha / \beta$ in Gy is from the linearquadratic model.

The BED is calculated as given in Fowler [14] as

$$
B E D=(n d) \times\left(1+\frac{d}{\alpha / \beta}\right)
$$

The single fraction equivalent dose (SFED) is obtained from the BED by substituting $\mathrm{d}=\mathrm{SFED}$ and $n=1$, and then solving for SFED giving the following equation:
Table 1 BED and SFED calculated for three hypothetical $\alpha / \beta$ ratios for the four dose regimes used. The fraction number ( $n)$, dose per fraction (d), biologically effective dose (BED), and single fraction equivalent dose (SFED) are all included

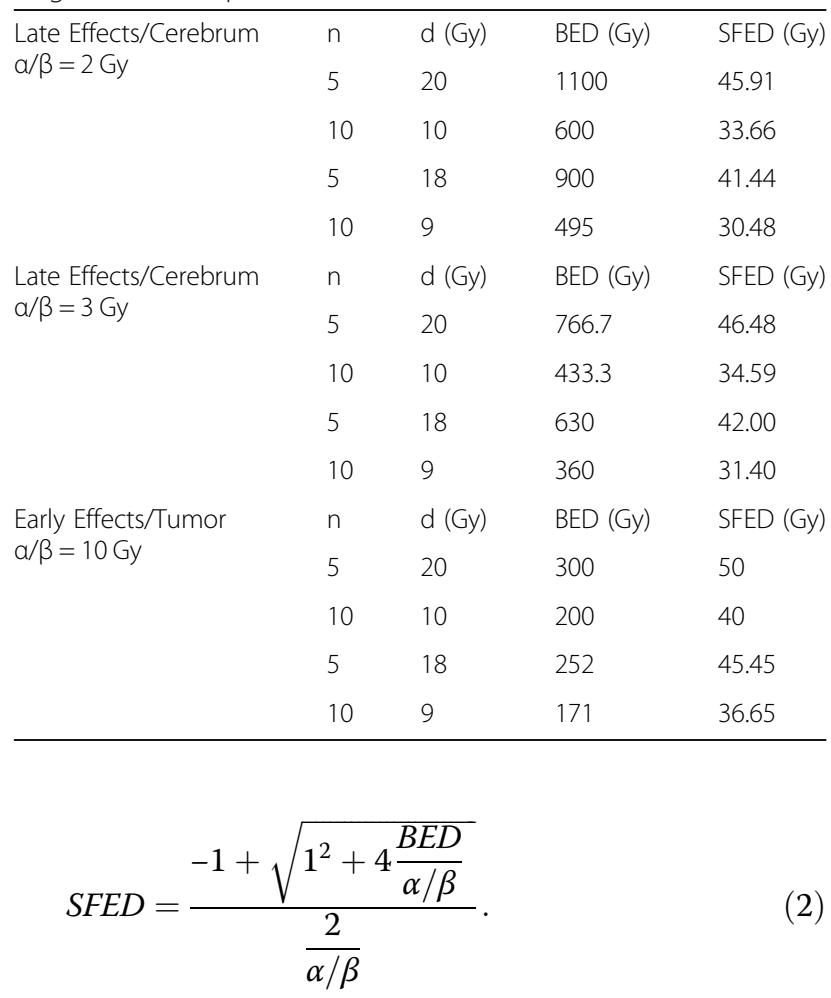

\section{Magnetic resonance imaging (MRI)}

Inhaled isoflurane was used to anaesthetize mice prior to imaging. Mice received an intraperitoneal injection of $0.2 \mathrm{~mL}$ of Multihance (gadobenate dimeglumine; Bracco Diagnostics Inc., Princeton, NJ) prior to imaging diluted to a 1:10 ratio in saline. A Bruker BioSpec 70/30USR $7 \mathrm{~T}$ MRI (Billerica, MA) was used to image mice at multiple timepoints up to a final timepoint of six weeks. RARE T2-weighted images (Effective $\mathrm{TE}=40 \mathrm{~ms}$, TR $=4000$ ms, Averages $=4)$ and MSME T1-weighted images (TE = $8 \mathrm{~ms}, \mathrm{TR}=500 \mathrm{~ms}$, Averages $=4$ ) were acquired. Twenty one slices with a $0.5 \mathrm{~mm}$ slice thickness were obtained for each scan type with the 3rd slice of both set of scans centered on where the olfactory bulbs and the rest of the cerebrum were separated. The matrix size of the scans was 128 pixels by 128 pixels with a field size of 15 by $15 \mathrm{~mm}^{2}$, with a corresponding resolution of $\sim 0.117$ $\mathrm{mm}$.

\section{MRI data analysis}

Radiation necrosis lesion quantification was assessed using a semi-automatic threshold segmentation algorithm as we have previously performed in this model [13]. Lesion is defined as regions of hyperintensity and hypointensity within the brain in this study with $\mathrm{T} 1$ and $\mathrm{T} 2$ images 
being analyzed independently. Both the upper and lower thresholds were chosen to be two standard deviations from the mean in normal mice. Brain segmentation and defining of lesion was carried out with a MATLAB (MathWorks, Natick, MA) program written in-house. Once the algorithm determined which voxels comprised the lesion, the lesion volumes is calculated by multiplying the total number of voxels by the unit volume for a voxel based on the scan geometry. In our scans, each voxel has a unit volume of roughly $0.007 \mathrm{~mm}^{3}$.

\section{Statistics}

Quantitative data were compiled in Prism 8 (GraphPad Software, San Diego, CA) for the generation of plots and statistical analysis. When summary statistics are presented, data are shown as mean \pm standard deviation. Two-Way ANOVA with a Tukey post-hoc test was used to compare the lesion volumes as a function of radiation scheme and time for T1 and T2 images independently.

\section{Histology}

Mice were euthanized after final imaging with their brains collected and left fixed in $4 \%$ paraformaldehyde with graded alcohols being used for processing. Mouse brains were embedded in paraffin. Hematoxylin and eosin (H\&E) staining was used on four micrometer sections of each mouse brain. An Evos XL (Life Technologies, Carlsbad, CA) digital inverted microscope was used to evaluate and photograph brain sections.

\section{Results}

The original purpose of our study was to create a fractionated mouse model of radiation necrosis that we could compare to our single fraction model. Mice were irradiated with four fractionation schemes and tracked up to 6 weeks with lesion volumes being measured. The lesion volumes measured were compared to what is generated with the same irradiation setup but single fraction doses [13]. Lesion development is observable on MRI in mice irradiated with all four fractionation schemes that is similar to what is observed in single fraction irradiations of 80 Gy or higher as is seen in Fig. 1 (Panels a and b). The two $100 \mathrm{~Gy}$ total fractionation schemes of 5 fractions of 20 Gy and 10 fractions of 10 Gy had lesion volumes most comparable to what is observed in single fraction irradiations of $90 \mathrm{~Gy}$, while the two $90 \mathrm{~Gy}$ total fractionation schemes of 5 fractions of $18 \mathrm{~Gy}$ and 10 fractions of $9 \mathrm{~Gy}$ had lesion volumes most comparable to what is observed in single fraction irradiations of 80 Gy. Using a Two-Way ANOVA, there was no significant difference between 5 fractions of 20 Gy and 1 fraction of $90 \mathrm{~Gy}, 5$ fractions of $18 \mathrm{~Gy}$ and 1 fraction of $80 \mathrm{~Gy}$, and 10 fractions of $9 \mathrm{~Gy}$ and 1 fraction of $80 \mathrm{~Gy}$ at either 4 or 6 weeks post-irradiation on both T2 and T1. 10 fractions of $10 \mathrm{~Gy}$ and 1 fraction of $90 \mathrm{~Gy}$ showed a significant difference at 4 weeks post-irradiation on T2 (Tukey $P=0.0282$ ) and T1 (Tukey $P=0.0492$ ), but did not show a significant difference 6 weeks post-irradiation. Similarly, quantification of the radiation necrosis lesion on based on hematoxylin and eosin staining found no differences between the irradiation schemes at 6 weeks post-irradiation (Fig. 1 Panel c). A one-way ordinary ANOVA of the Histology Grade vs Dose (Gy) data found that the most significant Tukey post-hoc adjusted $p$ value was 0.0617 between $20 \mathrm{~Gy}$ * 5 vs. $80 \mathrm{~Gy}$, showing a lack of statistical significance in this data.

Furthermore, brain swelling is observable in the former two fractionation schemes but mostly absent in the latter two fractionation schemes as observed in Fig. 2 with measurable damage caused by radiation on both
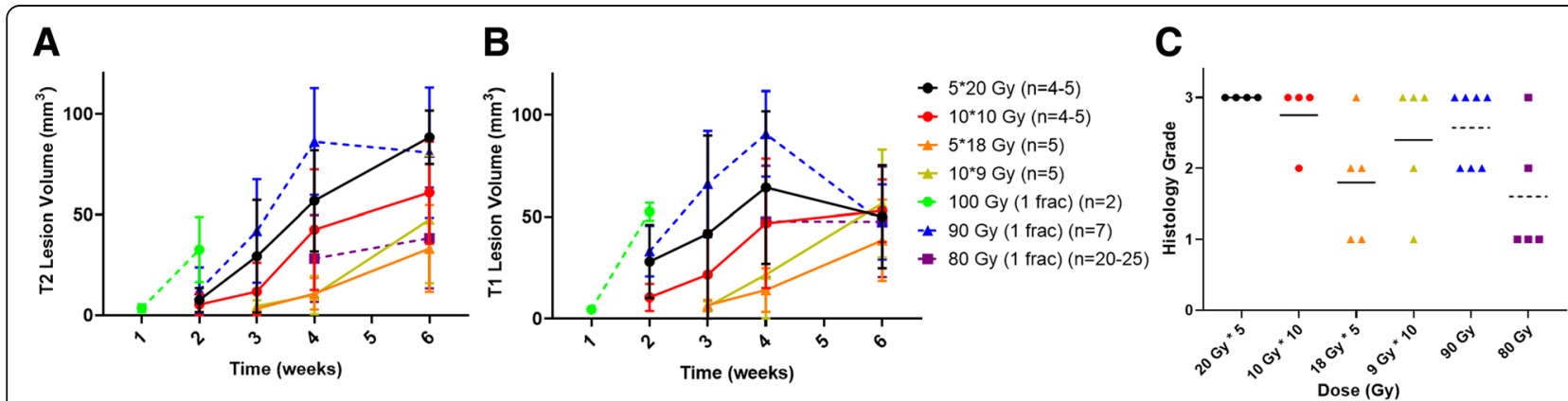

Fig. 1 Lesion progression as a function of time post-irradiation. The figure shows the lesion size (in $\mathrm{mm}^{3}$ ) over time (in weeks) for mice that received multiple fractionation regiments as well as single fraction doses between 80 and 100 Gy for T2-Weighted (Panel a) and post-contrast T1weighted (Panel b) MRI images as well as histological scores at 6 weeks post-irradiation (Panel $\mathbf{c}$ ). The data is presented as mean \pm standard deviation for lesion progression in Panels $\mathbf{a}$ and $\mathbf{b}$ with mean values present as bars in Panel $\mathbf{c}$. The number of animals in each group (n) ranges from 2 to 25 . Notice that the fractionated schemes' lesion volumes are comparable to those of the three single fraction regiments against the expectations of the SFED seen in Table 1. Also, the post-radiation side effects for single fraction 100 Gy irradiations were severe enough that mice needed to be sacrificed at 2 weeks with data not available past this point. Histological scores for $100 \mathrm{~Gy}$ are not in Panel c since post-irradiation side effects required sacrificing these mice early at 2 weeks 


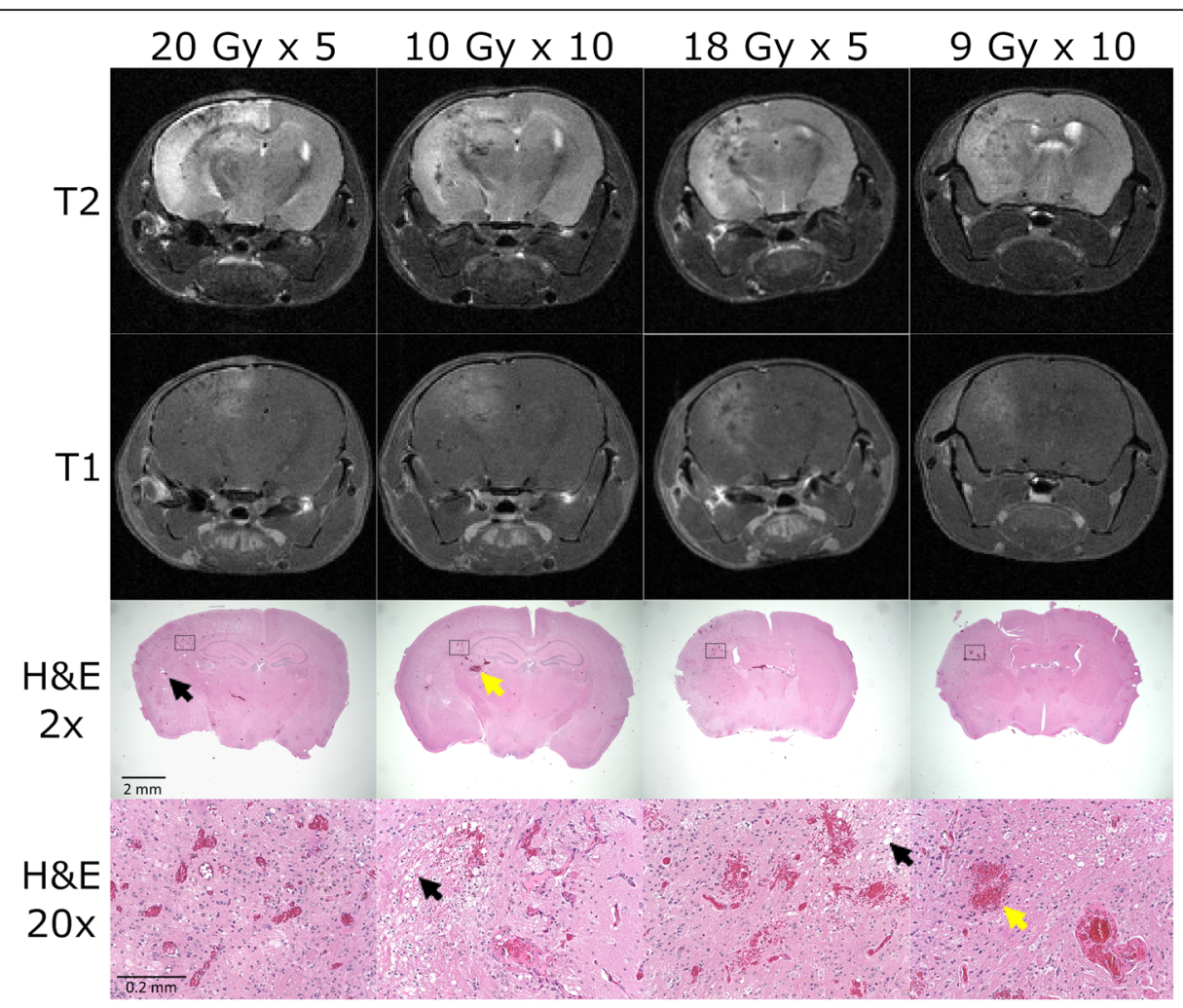

Fig. 2 Representative MRI and histology images of murine radiation necrosis. T2-weighted (1st row), post-contrast T1-weighted ( 2 nd row), and H\&E images with a magnification of 2 and 20 respectively (3rd and 4th row) are presented for all four fractionation schemes: 5 fractions of 20 Gy, 10 fractions of $10 \mathrm{~Gy}, 5$ fractions of $18 \mathrm{~Gy}$, and 10 fractions of $9 \mathrm{~Gy}$. The $3 \mathrm{rd}$ and 4 th H\&E images have black scales bars equal to $2 \mathrm{~mm}$ and 0.2 $\mathrm{mm}$ respectively. Areas of radiation injury on MRI (left hemisphere) correspond to visible pathology such as interstitial edema (black arrows) and hemorrhage (yellow arrows) on H\&E images

MRI and Hematoxylin and eosin (H\&E) being generally greater in the former than the latter. The results thus show that fractionation is less effective at controlling lesion development than expected with total dose being an important predictive factor of lesion development.

\section{Discussion}

The purpose of this study was to create a fractionated mouse model of radiation necrosis that could be compared to our single-fraction model. All four fractionation schemes had single fraction equivalent doses less than 50 Gy. Though our single fraction irradiations of $50 \mathrm{~Gy}$ did not produce radiation necrosis within 26 weeks [13], all fractionated regimes led to measurable radiation necrosis on MRI and histology. The two 100 Gy total fractionation schemes had lesion volumes most similar to single fraction irradiations of $90 \mathrm{~Gy}$, while the two $90 \mathrm{~Gy}$ total fractionation schemes were most similar to single fraction irradiations of $80 \mathrm{~Gy}$.

The lack of sparing expected by fractionation observed is surprising, as this has been well evidenced in most tissues in laboratory animals leading to fractionation as the most common way we perform radiotherapy [15-18]. However, the sparing effects of radiation being less than anticipated in the rodent brain is not a new phenomenon. A prior report on a murine model of radiation necrosis [8] showed no difference when comparing a 60 Gy (50\% isodose) treatment given in both 1 and 3 fractions. Similar results have been found when comparing fractionated to unfractionated regimes in a rat model of cognitive impairment [19] with single-fraction doses ranging from 11 to $17 \mathrm{~Gy}$.

One potential explanation for the lack of sparing is the large fraction sizes and total dose in our study. However, various hypo-fractionated schemes are reported for lateresponding tissues with similar fraction sizes to the low end of what we used which generally conform to the $\operatorname{BED}[7,20,21]$. Another potential reason may be that we did not wait long enough in between delivering fractions. A prior report [22] gives a repair halftime for radiation necrosis to be $38.1(6.9-76)$ hours based on human data. Thus, irradiating every other day instead of every day may result in additional sparing of damage.

An important limitation of our work is the rodent brain itself. The differences in levels of brain folding and white to grey matter composition between rodents and humans complicates the interpretations of any findings. 
Rodent models of radiation induced brain injury may not behave in a manner consistent with human disease. A clear example of this is the large doses that are needed to generate radiation necrosis in rodents [13]. We have also seen this in the past with models of radiation induced cognitive impairment not replicating the MRI deficits seen in humans [23]. Fractionation may not be as important a parameter in our murine model compared to parameters such as total dose, but we believe this is much more likely a feature of rodent brain irradiation models that is unlikely to be reflected in human patients.

\section{Abbreviations}

BED: Biologically effective dose; H\&E: Hematoxylin and eosin; MRI: Magnetic resonance imaging; SFED: Single fraction equivalent dose; $\alpha / \beta$ : alpha/beta ratio

\section{Acknowledgements}

We would like to thank the following Purdue core facilities where the work was performed: Purdue Small Animal MRI facility and Histology Research Lab. We also wish to thank Dr. Chang-Deng Hu for providing access to the X-Rad 320

\section{Authors' contributions}

AJB helped design and performed the experiments, analyzed the data, and wrote the manuscript. CJPT designed experiments and wrote and edited the manuscript. Both authors read and approved the final manuscript.

\section{Funding}

There are no funding sources to report for this study.

\section{Availability of data and materials}

The datasets used and/or analysed during the current study are available from the corresponding author on reasonable request.

\section{Ethics approval}

All animal experiments were approved by the Purdue Animal Care and Use Committee.

\section{Consent for publication}

Not applicable

\section{Competing interests}

The authors declare that they have no competing interests.

Received: 11 March 2019 Accepted: 7 August 2019

Published online: 13 August 2019

\section{References}

1. Baskar R, Lee KA, Yeo R, Yeoh K-W. Cancer and radiation therapy: current advances and future directions. Int J Med Sci. 2012;9(3):193-9.

2. Nahum AE. The radiobiology of hypofractionation. Clin Oncol R Coll Radiol G B. 2015;27(5):260-9.

3. Mehta S, Suhag V, Semwal M, Sharma N. Radiotherapy: basic concepts and recent advances. Med J Armed Forces India. 2010;66(2):158-62.

4. Morgan TM, Zaenger D, Switchenko JM, Eaton BR, Crocker IR, Ali AN, et al. Fractionated radiotherapy is associated with lower rates of treatmentrelated edema than stereotactic radiosurgery in magnetic resonance imaging-defined Meningiomas. World Neurosurg. 2019;121:e640-6.

5. Cozzarini C, Fiorino C, Deantoni C, Briganti A, Fodor A, La Macchia M, et al. Higher-than-expected severe (grade 3-4) late urinary toxicity after postprostatectomy hypofractionated radiotherapy: a single-institution analysis of 1176 patients. Eur Urol. 2014;66(6):1024-30.

6. Fiorino C, Cozzarini C, Rancati T, Briganti A, Cattaneo GM, Mangili P, et al. Modelling the impact of fractionation on late urinary toxicity after postprostatectomy radiation therapy. Int J Radiat Oncol Biol Phys. 2014; 90(5):1250-7.
7. Kirkpatrick JP, Soltys SG, Lo SS, Beal K, Shrieve DC, Brown PD. The radiosurgery fractionation quandary: single fraction or hypofractionation? Neuro-Oncol. 2017;19(2):ii38-49.

8. Jiang $X$, Yuan L, Engelbach JA, Cates J, Perez-Torres CJ, Gao F, et al. A gamma-knife-enabled mouse model of cerebral single-hemisphere delayed radiation necrosis. PLoS One. 2015;10(10):e0139596.

9. Perez-Torres CJ, Yuan L, Schmidt RE, Rich KM, Drzymala RE, Hallahan DE, et al. Specificity of Vascular Endothelial Growth Factor Treatment for Radiation Necrosis. Radiother Oncol J Eur Soc Ther Radiol Oncol. 2015;117(2):382-5.

10. Perez-Torres CJ, Engelbach JA, Cates J, Thotala D, Yuan L, Schmidt RE, et al. Toward Distinguishing Recurrent Tumor from Radiation Necrosis: DWI and MTC in a Gamma Knife ${ }^{\circledast}$ Irradiated Mouse Glioma Model. Int J Radiat Oncol Biol Phys. 2014;90(2):446-53.

11. Constanzo J, Masson-Côté L, Tremblay L, Fouquet JP, Sarret P, Geha S, et al. Understanding the continuum of radionecrosis and vascular disorders in the brain following gamma knife irradiation: An MRI study. Magn Reson Med. 2017;78(4):1420-31.

12. Kondo N, Sakurai Y, Takata T, Takai N, Nakagawa Y, Tanaka H, et al. Localized radiation necrosis model in mouse brain using proton ion beams. Appl Radiat Isot Data Instrum Methods Use Agric Ind Med. 2015;106:242-6.

13. Boria AJ, Perez-Torres CJ. Influence of dose uniformity when replicating a gamma knife mouse model of radiation necrosis with a preclinical irradiator. Radiat Res. 2019;191(4):352-9.

14. Fowler JF. The linear-quadratic formula and progress in fractionated radiotherapy. Br J Radiol. 1989;62(740):679-94.

15. Park $\mathrm{S}, \mathrm{Urm} \mathrm{S}, \mathrm{Cho} \mathrm{H}$. Analysis of biologically equivalent dose of stereotactic body radiotherapy for primary and metastatic lung tumors. Cancer Res Treat Off J Korean Cancer Assoc. 2014;46(4):403-10.

16. Jones B, Dale RG, Deehan C, Hopkins Kl, Morgan DA. The role of biologically effective dose (BED) in clinical oncology. Clin Oncol R Coll Radiol G B. 2001; 13(2):71-81.

17. Lee C-C, Yen C-P, Xu Z, Schlesinger D, Sheehan J. Large intracranial metastatic tumors treated by gamma knife surgery: outcomes and prognostic factors. J Neurosurg. 2014;120(1):52-9.

18. Urbanski K, Gasinska A, Pudelek J, Fowler JF, Lind B, Brahme A. Biologically effective doses in radiotherapy of cervical carcinoma*. Neoplasma. 2004; 51(3):228-38.

19. Greene-Schloesser DM, Kooshki M, Payne V, Jr RBD, Wheeler KT, MethenyBarlow LJ, et al. Cellular response of the rat brain to single doses of 137Cs Y rays does not predict its response to prolonged 'biologically equivalent' fractionated doses. Int J Radiat Biol. 2014;90(9):790-8.

20. van Baardwijk A, Tomé WA, van Elmpt W, Bentzen SM, Reymen B, Wanders $\mathrm{R}$, et al. Is high-dose stereotactic body radiotherapy (SBRT) for stage I nonsmall cell lung cancer (NSCLC) overkill? A systematic review. Radiother Oncol. 2012;105(2):145-9.

21. Daly ME, Perks JR, Chen AM. Patterns-of-Care for Thoracic Stereotactic Body Radiotherapy among practicing radiation oncologists in the United States. J Thorac Oncol. 2013:8(2):202-7.

22. Bender ET. Brain necrosis after fractionated radiation therapy: is the halftime for repair longer than we thought? Med Phys. 2012;39(11):7055-61.

23. Rancilio NJ, Dahl S, Athanasiadi I, Perez-Torres CJ. Design, construction, and in vivo feasibility of a positioning device for irradiation of mice brains using a clinical linear accelerator and intensity modulated radiation therapy. Int J Radiat Biol. 2017;93(12):1321-6.

\section{Publisher's Note}

Springer Nature remains neutral with regard to jurisdictional claims in published maps and institutional affiliations. 